\title{
TrataMENTO CIRÚRGICO VIDEOLAPAROSCÓPICO DA DOENÇA DO REFLUXO GASTROESOFAGIANO: TÉCNICA DE NISSEN MODIFICADA - RESULTADOS CLÍNICOS E FUNCIONAIS
}

\author{
L. R. Lopes, N. A. Brandalise, N. A. Andreollo, L. S. Leonardi \\ Trabalhodesenvolvidono Grupo deCirurgiadoEsôfago-Estômago-DuodenodaDisciplinadeMoléstiasdoAparelho \\ Digestivo daFaculdade deCiências Médicas daUniversidadeEstadual deCampinas, SP.
}

RESUMO - A doença do refluxo gastroesofagiano é muito freqüente na população e o tratamento cirúrgico é indicado em um número respeitável de pacientes. A escolha da via de acesso é a laparoscopia. Várias técnicas anti-refluxo podem ser empregadas e preferimos a técnica de Nissen modificada.

Овjetivo. Analisar os parâmetros clínicos e funcionais préoperatórios comparados com os mesmos parâmetros pós-operatórios com a técnica empregada.

Métodos. Um grupo de 59 pacientes foi submetido a tratamento cirúrgico pela técnica de Nissen modificada videolaparoscópica. 0 diagnóstico pré-operatório foi feito por exame radiográfico contrastado e endoscopia digestiva alta em todos os pacientes. A manometria do esôfago realizada em 35 e a cintilografia em 15. Esofagite complicada ocorreu em 54,2\% sendo 21 pacientes $(35,6 \%)$ com epitélio de Barrett. A técnica cirúrgica laparoscópica foi concluída em todos os pacientes. 0 tempo médio de cirurgia foi de 123,9 minutos.

ResUltados. Não ocorreram complicações intra-operatórias. A alta se deu em média com 47,6 horas. Sintomas de disfagia, dor, epigastralgia, regurgitação e flatulência até $030^{\circ}$ dia ocorreram em $48,1 \%$ dos pacientes. Ocorreu uma reoperação por recurrência da doença e um óbito por necrose do fundo gástrico. 0 seguimento médio foi de 20,8 meses. Os exames pós-operatórios radiográficos, endoscópicos, manométricos e de cintilografia mostraram melhora significativa, bem como a avaliação clínica, que mostrou excelentes e bom resultados em $93,1 \%$ dos pacientes.

ConClusäo. A cirurgia de Nissen modificada videolaparoscópica corrigiu a doença do refluxo gastroesofagiano na maioria dos doentes acompanhados, associada à baixa morbimortalidade.

UnITERMOS: Refluxo gastroesofagiano. Laparoscopia. Cirurgia.

\section{INTRODUÇÃO}

Nos últimos anos, o surgimento da videocirurgia e da videolaparoscopia, como opção de acesso à cavidade abdominal sem a necessidade de grandes incisões, permitindo a realização da cirurgia com total reprodução do método consagrado na cirurgia aberta, constituiu-se no grande avanço da cirurgia nos anos que antecedem o novo século, alcançando grande sucesso entre cirurgiões, médicos e pacientes'.

Trata-se de método seguro, com resul-

$$
\begin{gathered}
\text { *Correspondência: } \\
\text { Prof.Dr.LuizRobertoLopes } \\
\text { RuaAméliaM. dePaulaVentrini,365-Cep: 13086-040 } \\
\text { Campinas-SP_lopes@obelix.unicamp.br }
\end{gathered}
$$

tados comparáveis aos da cirurgia aberta em muitos procedimentos, apresentando vantagens como diminuição da dor no pósoperatório, recuperação rápida, alta hospitalar precoce, reintegração às atividades diárias e ao trabalho em curto período de tempo e aspecto estético favorável, com mínima mudança no estilo de vida do paciente $^{2}$.

Em 199| foram publicadas as primeiras séries da cirurgia de Nissen para o tratamento da doença do refluxo gastroesofagiano, com o auxílio da videolaparoscopia,

Dallemagne et al., $(|99|)^{3}$, relataram os resultados em 12 pacientes operados pela técnica de Nissen, com sucesso em nove e três conversões (25\%), com 10\% de mor- bidade operatória. Os mesmos autores mostraram, dois anos depois, os resultados de 132 pacientes operados pela mesma técnica, sem mortalidade, 3,3\% de conversão e morbidade de 7,5\% $\%^{5}$. Assim foi demonstrada a exeqüibilidade da técnica e outros trabalhos repetiram ou até melhoraram os resultados, atestando a segurança do procedimento 6 .

É importante assinalar que o procedimento básico do tratamento cirúrgico da doença do refluxo gastroesofagiano não foi alterado, sendo reproduzida a mesma cirurgia convencionalmente aceita como a de escolha ${ }^{6-9}$, mantendo o pressuposto de que faz parte do desejo do cirurgião dispor de técnica eficiente, segura e pouco traumáti- 
$\mathrm{ca}^{10}$, aplicando o melhor acesso para a operação adequada, sem cair na tentação de buscar a melhor operação para o acesso" ".

Apesar do curto tempo de avaliação dos pacientes submetidos a cirurgia para correção do refluxo gastroesofagiano, na maioria das comunicações podemos admitir que os resultados são comparáveis aos resultados anteriormente obtidos pela técnica aberta, acrescido das vantagens proporcionadas pela abordagem laparoscópica ${ }^{12}$.

O objetivo deste trabalho é mostrar os resultados clínicos e funcionais obtidos com a cirurgia de Nissen modificada realizada por videolaparoscopia.

\section{Métodos}

No período de junho de 1994 a dezembro de 1996, 59 pacientes consecutivos, portadores de DRGE, foram submetidos a tratamento cirúrgico por videolaparoscopia e acompanhados prospectivamente, sendo 32 (54,2\%) do sexo feminino. A idade variou de 15 a 82 anos (média de 52,6 anos). $\mathrm{O}$ peso variou de 40 a $91 \mathrm{Kg}$. O tempo de sintomas de refluxo variou de 12 a 480 meses.

A indicação da cirurgia foi baseada na história clínica e nos exames complementares diagnósticos: estudo contrastado do esôfago-estômago-duodeno, endoscopia digestiva alta, eletromanometria do esôfago e pesquisa do refluxo gastroesofagiano por cintilografia. Os resultados dos exames préoperatórios foram comparados com os resultados pós-operatórios. A ultra-sonografia do abdome foi realizada em 40 pacientes para avaliação de outras doenças, principalmente a da vesícula biliar, que foi encontrada em 9 (15,5\%) pacientes. Todos os pacientes haviam sido tratados clinicamente sem melhora por um período de tempo variável de 6 meses a 15 anos. Três pacientes tinham diagnóstico prévio de esclerodermia.
O diagnóstico final pré-operatónio após a endoscopia digestiva alta baseado na classificação da esofagite proposta por Savary-Miller ${ }^{3}$ é apresentado na Tabela I.

Assim, 27 doentes (45,8\%) apresentavam esofagite não complicada e 32 doentes $(54,2 \%)$ esofagite complicada, dentre o quais 21 (35,6\%) com mucosa de Barrett.

\section{Técnica de Nis- sen modificada}

Após preparo habitual pré-operatório, anestesia geral intravenosa e inalatória com ventilação controlada mecânica e monitoramento obrigatório, os pacientes foram posicionados segundo a técnica européia. A equipe cirúrgica e os equipamentos seguiram a distribuição conhecida, assim como a realização do pneumoperitônio e a colocação dos trocartes (Figura I).

A cirurgia foi iniciada com a dissecção da transição esofagogástrica, isolamento do esôfago abdominal, reparo do mesmo com penrose fino, dissecção dos braços do pilar direito do diafragma, liberação do fundo gástrico das aderências frouxas do baço e do retroperitônio, sem ligadura ou secção dos vasos breves na maioria dos pacientes.

Após testar a possibilidade de realizar a fundoplicatura sem tensão, os braços do pilar foram aproximados com pontos separados em X com fio inabsorvível e nó interno (policron 2-0, agulha atraumática de
$2,5 \mathrm{~cm}$ ). Foi deixado um espaço de uma pinça de apreensão aberta entre o esôfago e a aproximação do pilar.

A fundoplicatura foi realizada com a passagem do fundo gástrico por detrás do esôfago, sendo o primeiro ponto total, e os demais aproximando o estômago ao esôfago de maneira parcial, em número aproximado de quatro pontos, sendo dois acima de ambos os lados do esôfago e dois abaixo da mesma maneira em relação ao primeiro. Tomou-se cuidado para manter a válvula frouxa ao redor do esôfago (Figura 2).

\section{Cuidados pós-operatórios}

Não foi utilizado antibiótico profilático e o jejum foi mantido por até 24 horas. A analgesia foi feita nas primeiras horas e depois quando solicitado. A alimentação foi 
introduzida no primeiro pós-operatório à base de líquidos e a alta hospitalar quando o paciente estava sem dor ou vômitos. 0 paciente foi orientado a se alimentar com dieta leve nas 3 ou 4 semanas subseqüentes. $O$ retorno ambulatorial se deu com sete dias para a retirada dos pontos, com 30 dias para uma avaliação inicial, e após o quarto mês quando foram solicitados os exames de controle.

\section{Análise estatística}

Para a análise estatística das diferentes variáveis foram utilizados o teste não paramétrico de McNemar, o teste de Wilcoxon, o teste do $X^{2}$ de Stuart-Maxwell e o teste de Fischer.

\section{Resultados}

A técnica proposta de Nissen modificada foi realizada por videolaparoscopia em todos os pacientes sem nenhuma conversão para cirurgia aberta, com tempo de cirurgia variando de 60 a 240 minutos (I23,9 minutos). O acesso à cavidade abdominal por laparoscopia permitiu ainda tratar 9 (I5,5\%) pacientes desta série com colecistopatia crônica calculosa. Três pacientes tinham diagnóstico de esclerodermia em fases avançadas, com esofagite graus II e IV e muita sintomatologia, sendo operados da mesma forma. A opção foi pela cirurgia conservadora obtendo melhora clínica da sintomatologia e do grau de esofagite ao exame endoscópico.

Não ocorreram complicações intraoperatórias graves, exceto dois pacientes que apresentaram enfisema subcutâneo e dois com aumento da concentração de CO2 expirado.

Um paciente apresentou choque séptico no pós-operatório imediato com sinais de peritonite e, à laparotomia exploradora, encontrou-se necrose parcial de 3 por $4 \mathrm{~cm}$ no fundo gástrico usado para confecção da válvula. Apesar da ressecção da área e do tratamento instituído o paciente veio a óbito. A alta hospitalar ocorreu entre 24 e 220 horas com média de 48 horas.

Dos sintomas imediatos até $030^{\circ}$ dia de pós-operatório, a disfagia ocorreu em $22,4 \%$, sendo necessário realizar endoscopia em três deles. A melhora ocorreu gradati-

vamente. Seis pacientes (I2\%) que foram dilatados no pré-operatório por apresentarem estenose, foram também dilatados no pós-operatório, e apenas dois continuaram posteriormente em programa de dilatação. Um destes pacientes necessitou ser reoperado por manter esofagite grave e estenose, sendo submetido à gastrectomia Y-Roux e vagotomia troncular. Apresenta-se com melhora da sintomatologia, mantendo ainda as dilatações endoscópicas do esôfago.

O retorno às atividades laborativas, exceto trabalho pesado, deu-se em média com 15 dias. $O$ tempo de seguimento dos pacientes variou de 5 a 37 meses, com média de 20,8 meses.

Os sintomas clínicos mais significativos, referidos no pré-operatório, foram comparados no pós-operatório a partir do quarto mês de cirurgia com melhora acentuada e foram: pirose retroesternal, regurgitação e disfagia. Os testes estatísticos realizados mostraram diferenças significativas $(p<0.05)$ para os sintomas mais importantes relatados entre o pré e o pós-operatório.

Tomando-se como base a classificação de Visick modificada, verificou-se que 54 $(93,1 \%)$ pacientes apresentaram resultados excelentes e bons, enquanto que quatro (6,9\%) pacientes não apresentaram melhora clínica.
Comparando-se o resultado do grau de esofagite pré-operatório pela classificação de Savary-Miller, dividido em não complicada (Graus I, II e III) e complicada (Grau IV), em relação ao resultado clínico pós-operatório pela classificação de Visick modificada (I e II satisfeitos e III e IV não satisfeitos), verificou-se que $96,3 \%$ dos pacientes com esofagite não complicada estavam satisfeitos com a cirurgia, enquanto que $90,3 \%$ dos pacientes com esofagite complicada também estavam satisfeitos com a cirurgia, não sendo diferença estatisticamente significativa.

O estudo radiográfico contrastado foi realizado em 59 pacientes no pré-operatório e em 39 pacientes no pós-operatório e os testes estatísticos realizados mostraram diferenças não significativas $(p>0.05)$ para os achados de estenose, úlcera e outros, e mostraram diferenças significativas $(p<0.05)$ para os achados de hérnia hiatal, esofagite, refluxo e alteração motora, entre o pré e o pós-operatório. Foram encontrados dois pacientes com hérnia paraesofagiana, correspondendo a 5, $1 \%$ dos exames realizados.

O estudo endoscópico foi realizado em 59 pacientes no pré-operatório e em 53 pacientes no pós-operatório e os testes estatísticos realizados mostraram diferenças não 
LOPES LR ET AL.

significativas $(p>0.05)$ para os achados de Barrett, refluxo, úlcera e outros, e mostraram diferenças significativas $(p<0.05)$ para os achados de hérnia hiatal, esofagite e estenose, entre o pré e o pós-operatório. Foram encontrados três pacientes com hérnia paraesofagiana, correspondendo a 5,6\% dos exames realizados.

O diagnóstico final pós-operatório do grau de esofagite pela EDA, segundo a classificação de Savary-Miller, em 53 exames realizados encontra-se na Tabela 2.

Comparando-se o grau de esofagite entre o pré-operatório e o pós-operatório, foi observada a seguinte distribuição apresentada na Tabela 3.

O estudo manométrico foi realizado no pré-operatório em 35 pacientes e no pósoperatório em 34 pacientes, observandose a pressão do EIE (Gráfico I) e as ondas motoras do corpo do esôfago. Foram encontrados os seguintes dados:

No pré-operatório, a pressão do EIE variou de 0 a $10 \mathrm{~mm} / \mathrm{Hg}$, com média de 4,8 $\mathrm{mm} / \mathrm{Hg}$ e mediana de $5 \mathrm{~mm} / \mathrm{Hg}(4,8+/$ 2,6) e no pós-operatório a variação foi de 4 a $25 \mathrm{~mm} / \mathrm{Hg}$, com média de $11,8 \mathrm{~mm} / \mathrm{Hg}$ e mediana de $10 \mathrm{~mm} / \mathrm{Hg}(\mid \mathrm{I}, 8+/ 4,6)$.

Os testes estatísticos realizados mostraram diferenças significativas $(p<0.05)$ para as medidas de pressão do EIE ao exame manométrico entre o pré e o pósoperatório.

Foram observadas alterações motoras do corpo esofagiano ao exame manométrico, em 17 pacientes (48,6\%) no préoperatório, e em II pacientes (32,4\%) no pós-operatório. Os testes estatísticos realizados mostraram diferenças não significativas $(p>0.05)$ para as alterações motoras do corpo do esôfago entre o pré e o pósoperatório.

O estudo cintilográfico foi realizado em I 5 pacientes no pré-operatório e 40 pacientes no pós-operatório, com o achado de $86,7 \%$ de refluxo no pré-operatório e $65 \%$ no pós-operatório. Os testes estatísticos realizados mostraram diferenças não significativas $(p>0.05)$ para 0 estudo do refluxo gastroesofagiano entre o pré e o pós-operatório.

\section{Discussão}

O tratamento definitivo para a cura da doença do refluxo gastroesofagiano tem sido a cirurgia, e a videocirurgia permitiu que esta opção ressurgisse, já que, por décadas, ela foi a melhor opção ${ }^{14}$. Trata-se de uma nova via de acesso para velhas operações onde os conceitos e princípios da cirurgia gastrointestinal dos anos passados não devem ser perdidos na corrente do entusiasmo atual|'5. Ela tornou-se o tratamento mais efetivo para mudar a história natural da doença e deve ser indicada segundo os mesmos critérios adotados anteriormente ${ }^{16,17}$.

Uma vez havendo indicação para o tratamento cirúrgico, a via de acesso preferencial deve ser a laparoscopia empregando-se as técnicas já conhecidas e aceitas como as melhores, quer sejam fundoplicaturas totais ou parciais, levando a crer que os resultados
Tabela 2 - Grau de esofagite no pós-operatório dos doentes operados pela técnica de Nissen modificada videolaparoscópica

$\begin{array}{ccc}\text { Grau de esofagite } & \text { Pacientes } & \text { Porcentagem } \\ \text { Sem } & 45 & 84,91 \% \\ \text { I } & 5 & 9,43 \% \\ \text { II } & 1 & 1,89 \% \\ \text { II } & 0 & 0,00 \% \\ \text { IV } & 2 & 3,77\end{array}$

Tabela 3 - Grau de esofagite no pré e no pós-operatório dos doentes operados pela técnica de Nissen modificada videolaparoscópica.

\begin{tabular}{ccr}
\hline Grau de esofagite & Pré-operatório & Pós-operatório \\
Sem & $0(0,00 \%)$ & $45(84,91 \%)$ \\
I & $7(11,90 \%)$ & $5(9,43 \%)$ \\
II & $\mid 1(18,60 \%)$ & $\mid(1,89 \%)$ \\
III & $9(15,30 \%)$ & $0(0,00 \%)$ \\
IV & $32(54,20 \%)$ & $2(3,77)$ \\
Total & $59(100,00 \%)$ & $53(100,00 \%)$ \\
\hline
\end{tabular}

Gráfico I - Resultados da manometria (pressão do esfíncter inferior esôfago em $\mathrm{mm} / \mathrm{Hg}$ ) para 31 pacientes pré e pós-cirurgia de Nissen modificada videolaparoscópica.

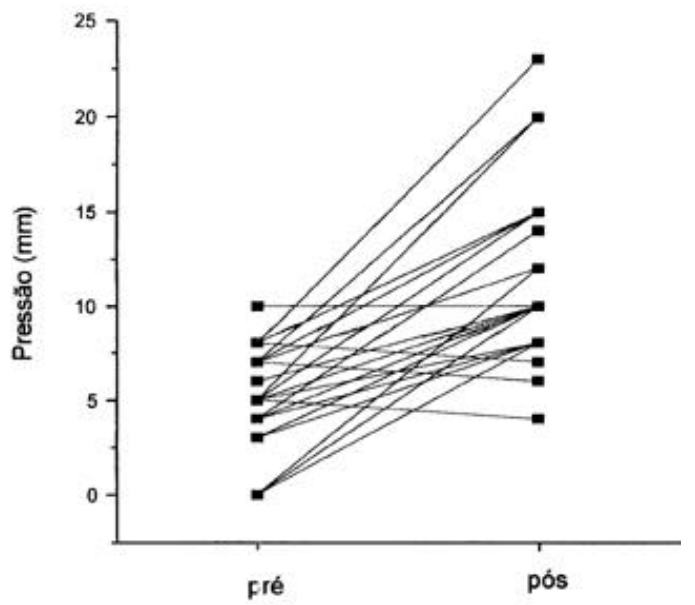

clínicos se repetirão $0^{5,12,19}$. Os resultados iniciais com a laparoscopia para o tratamento da DRGE, apesar do acompanhamento curto, foram encorajadores e sugerem que os resultados obtidos possam ser comparáveis àqueles da cirurgia aberta ${ }^{20}$, podendo reproduzir exatamente os resultados dos 
procedimentos cirúrgicos abertos ${ }^{21}$.

Os 59 pacientes desta casuística foram avaliados clinicamente e com exames subsidiários. Os exames subsidiários básicos seriam o RXEED e a EDA, enquanto que a MANO, a pHMETRIA e a CINTI teriam o seu uso seletivo nos pacientes com sintomas atípicos, disfagia, alterações motoras ao $R x$, sintomas típicos de refluxo com EDA normal, falha na terapêutica clínica levando a complicação e falha em cirurgia anti-reflu$x O$ anterior $^{18,22}$.

A esofagite complicada grau IV foi encontrada em $54,2 \%$ dos pacientes e $35,3 \%$ deles apresentavam epitélio de Barrett, mostrando tratar-se de pacientes com indicação para cirurgia a muito mais tempo e só agora encaminhados para a cirurgia. Em outras casuísticas o grau de esofagite complicada grau IV, bem como a presença de epitélio de Barrett variou de $6 \%$ a $29 \%$ 6, $12,23,24$.

A técnica proposta de Nissen modificada foi realizada em todos os pacientes sem necessidade de conversão para cirurgía aberta. Na literatura a taxa de conversão varia de $1,3 \%$ a $\mid 4,2 \%$, $12,24-31$.

A posição do paciente, dos equipamentos de armário, dos trocartes utilizados e da equipe cirúrgica segue a técnica européia como a maioria dos cirurgiões $\operatorname{adotam}^{3,6,9,32}$

Não foi realizada de rotina a liberação do fundo gástrico com a secção dos vasos breves, pois foi possível fazer a fundoplicatura sem tensão usando a parede anterior ou posterior do fundo gástrico. Anvari \& Allen (1996)33, Watson et al. (1994) ${ }^{34}$, Luostarinen \& Isolauri $(1996)^{35}$, Watson et al., $(1996)^{36}$, não realizaram a secção dos vasos breves e não referiram sintomas adversos no pós-operatório. Já Weerts et al., $(1993)^{5}$, lincarbone et al., (1995) ${ }^{37} \mathrm{e}$ Richardson et al., $(1996)^{18}$, ao fazerem a secção dos vasos breves, tiveram a intenção de facilitar a mobilização do fundo gástrico.
Assim como na descrição original de Nis$\operatorname{sen}^{38}$ não era realizada a secção dos vasos breves, GEAGEA (1991) também não a realizou. A liberação do fundo gástrico com a secção ou não dos vasos breves deve ser avaliada no intra-operatório, devendo ou não ser realizada.

Foi considerado imprescindível a aproximação dos ramos do pilar diafragmático com o cuidado de não apertar muito o ponto para não causar disfagia no pós-operatório, assim como referiram Munro et al. $(1996)^{39}$, Brandalise \& Aranha $(1996)^{9} \mathrm{e}$ Paula (1997)6.

A técnica de Nissen modificada foi realizada sem dificuldade e mesmo facilitou o procedimento devido a manter a válvula montada para a confecção dos pontos parciais.

As fundoplicaturas totais ou parciais são os procedimentos mais empregados para o tratamento da DRGE, tanto por via aberta como por laparoscopia. As mesmas mostraram resultados excelentes e bons quando por via aberta acima de $85 \%$ dos pacientes tendo passado pelo crivo do tempo por mais de 10 anos de acompanhamento 5,40,41,42. Como por laparotomia eram acompanhadas de dor, morbidade de 8 a $22 \%$, mortalidade de 0 a I, $4 \%$, tempo de recuperação hospitalar e domiciliar prolongados, a cirurgia era muitas vezes protelada ao máximo ${ }^{7,43}$.

Com o advento da videocirurgia e os benefícios dela decorrentes, as fundoplicaturas passaram a ser realizadas reproduzindo as mesmas técnicas da cirurgia aber$\mathrm{ta}^{43}$. Na verdade, a cirurgia laparoscópica facilitou a exposição do campo operatório, permitindo uma visão mais detalhada e a possibilidade de uma cirurgia mais adequa$\mathrm{da}^{32,43}$.

O tempo cirúrgico nesta casuística manteve-se na média encontrada na literatu-

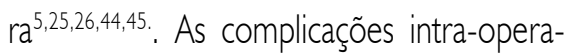
tórias observadas ocorreram em 3,3\% dos pacientes relacionadas ao pneumoperitônio (enfisema subcutâneo e hipercabia). As taxas de complicações relatadas variam de 0 a 10\% com enfisema subcutâneo, hipercabia, hemorragia, perfuração de esôfago ou estômago e pneumotórax. As lesões de baço encontradas na cirurgia aberta entre 2 a 8,5\% ${ }^{23,26}$ não são descritas pela via laparoscópica.

Ocorreu um óbito no pós-operatório imediato devido a necrose parcial do fundo gástrico. Apesar da reoperação e ressecção da área comprometida, o paciente faleceu. Tratava-se de um paciente de 72 anos de idade e que foi um dos poucos casos iniciais da casuística em que foi realizada a liberação do fundo gástrico com secção dos vasos breves com grampos metálicos. As muitas publicações relatam casos esporádicos de complicações graves semelhantes à es$\operatorname{sas}^{46,47,48}$, com taxas de mortalidade ao redor de $0,5 \%$, semelhantes às da cirurgia aberta $(2 \%)^{23}$.

O sintoma mais comum referido até o $30^{\circ}$ dia de pós-operatório foi a disfagia em $13(22,4 \%)$ pacientes. Seis deles com estenose e dilatação no pré-operatório necessitaram novas dilatações no pós-operatório.

A alta hospitalar ocorreu em média com 48 horas, sendo semelhante ao tempo encontrado na literatura entre 2,8 e 4 dias $^{3,5,24,26,29,42,48}$. Para diminuir a possibilidade de sintomas de disfagia no pós-operatório, todos os pacientes foram orientados a ingerir alimentos líquidos e pastosos por um período aproximado de 4 semanas ${ }^{6,34}$.

Houve necessidade de reoperação de um paciente $(1,7 \%)$ por manter esofagite grau IV e estenose após um ano da cirurgia, sendo realizado gastrectomia Y-Roux. O paciente era portador de seqüela de AVC e estenose importante no pré-operatório. Continua em acompanhamento endoscópico para dilatações do esôfago. As taxas de reoperações encontradas variam de I,5\% $\%^{23}$ a $7,7 \%^{48}$ em decorrência de reflu- 
xo recorrente, disfagia severa e hérnia paraesofagina.

A partir do quarto mês de pós-operatório, os pacientes foram reavaliados por entrevista clínica e por exames complementares. Os sintomas clínicos avaliados no pré e pós-operatório mostraram melhora acentuada, com diferença significativa. Aproximadamente $93 \%$ dos pacientes consideraram os resultados excelentes e bons, estando satisfeitos com a cirurgia (Visick I e II), em um tempo médio de acompanhamento de 20,8 meses. Dallemagne (1994) ${ }^{27}$ referiu 90,5\% de excelentes e bons resultados com a cirurgia de Nissen VLP em acompanhamento de 15,8 meses. Paluzzi (1997) ${ }^{49}$ referiu $96 \%$ de resultados de Visick I e II após a técnica de Nissen VLP em acompanhamento de 15 meses. Laws; Clements; Swillie (1997) ${ }^{50}$ observaram não haver diferença entre o grau de satisfação com a cirurgia de válvula parcial ou total por VLP, sendo acima de $90 \%$ de Visick I e II. A técnica de Nissen modificada obteve os mesmos resultados das técnicas clássicas pois mantém o princípio da fundoplicatura curta e frouxa, permitindo um resultado muito satisfatório. Além disso, os sintomas comuns observados de empachamento (15\%), impossibilidade de eructar e vomitar (63\%) e disfagia (14\%) $33,48,51,52$, foram encontrados nesta casuística em números menores (1,7\%,0\% e 3,4\%), respectivamente). Esses resultados foram imputados ao fato de ser realizada uma fundoplicatura que, por um lado é competente ao envolver o esôfago com um ponto completamente e, por outro lado, pode evitar os sintomas inconvenientes pela fundoplicatura parcial associada.

Os exames complementares radiográficos realizados mostraram melhora nos parâmetros analisados com diferença significativa entre o pré e o pós-operatório. Ficou evidenciado em dois pacientes $(5,1 \%)$ com hérnia paraesofagiana assin- tomáticos e mantidos em observação.

A endoscopia revelou uma melhora acentuada nos parâmetros analisados com diferença significativa entre o pré e o pósop. $O$ índice de cicatrização da esofagite alcançou 84,9\% dos pacientes. As taxas encontradas na literatura variam ao redor de $90 \%{ }^{24}$. Dos 15,1\% de pacientes que mostraram esofagite no pós-operatório, $75 \%$ deles tinham esofagite grau IV no préoperatório, mostrando com isso que o comprometimento do esôfago antes da cirurgia é fator importante para se alcançar resultados satisfatórios.

O epitélio de Barrett foi observado em 35,6\% dos pacientes. Para DeMeester et al. $(1990)^{53}$, a presença deste epitélio implica em estado avançado de comprometimento da DRGE, o que pode dificultar a obtenção de bons resultados com a cirurgia. Todos foram encaminhados para ablação deste epitélio com eletrocautério por endoscopia ${ }^{54}$. Em três pacientes $(5,6 \%)$ foi observado a presença de hérnia paresofagiana (duas vistas também no exame radiográfico), e, por estarem assintomáticos estão em observação. Sua ocorrência se deve a não aproximação dos braços do pilar ou a abertura do mesmo após fechamento, sendo sempre recomendado a aproximação na cirurgia anti-reflux ${ }^{55}$. A ocorrência varia em torno de $3 \% 6,56,57$. Perdikis et al. $(1997)^{58}$ recomendam a correção cirúrgica. Paula $(1997)^{6}$ descreveu 3\% de hérnia paraesofagiana e apenas uma reoperação por ter sintomas.

O estudo manométrico realizado demonstrou que a válvula criada com a técnica de Nissen modificada possibilitou um aumento da ZAP de $5 \mathrm{mmHg}$ para $1 \mathrm{I} \mathrm{mmHg}$ em média, sendo diferença significativa. Estes dados são comparáveis aos encontrados em pacientes submetidos à cirurgia de Nissen VLP6,814.

A avaliação cintilográfica mostrou também melhora do refluxo de $86,7 \%$ no pré para 65\% no pós-operatório, sem contudo constituir em diferença significativa. Este método isolado não permite o diagnóstico de certeza de refluxo sendo necessário correlacionar com os sintomas clínicos e outros exames.

\section{Conclusões}

A fundoplicatura laparoscópica pela técnica de Nissen modificada foi capaz de tratar a DRGE, apresentando vantagens adicionais em relação ao tratamento clínico prolongado e à cirurgia aberta. Vale ressaltar que 0 procedimento básico de tratamento não foi mudado, admitindo-se, assim, a possibilidade de se atingirem resultados, a longo prazo, semelhantes àqueles conseguidos pela cirurgia aberta tanto pela técnica de Nissen como pela técnica de Lind ${ }^{7}$. A efetividade da técnica laparoscópica não diferiu da técnica aberta, oferecendo vantagens, como tempo de hospitalização menor, tempo de recuperação mais rápido, pouca sintomatologia dolorosa com resultados cosméticos melhores ${ }^{60}$. A cirurgia anti-refluxo por laparotomia alcançou altos índices de alívio dos sintomas e, de maneira semelhante, a cirurgia por laparoscopia tem alcançado os mesmos resultados ${ }^{61}$, além de trazer os benefícios da cirurgia pouco invasiva. Podese observar que o método laparoscópico para o tratamento cirúrgico da DRGE foi seguro, desde que realizado por cirurgião experiente. O método possibilita a reprodução exata do mesmo procedimento rotineiramente empregado, evitando-se as desvantagens da cirurgia aberta e com as vantagens da cirurgia minimamente invasiva'. Portanto, houve mais vantagens do que desvantagens com a laparoscopia e, ainda, pode-se acreditar que os resultados funcionais a longo prazo sejam equivalentes aos obtidos com a cirurgia aberta'.

$\mathrm{Na}$ análise dos pacientes desta série, observou-se que, de maneira geral, a cirur- 
gia deve ser indicada nos pacientes com sintomas característicos e que não são controlados por medicamentos, associado à comprovação do diagnóstico pelo exame radiográfico contrastado do esôfago-estômago-duodeno para documentação de defeitos anatômicos e da endoscopia digestiva alta para documentação da esofagite. Os demais exames de eletromanometria do esôfago, cintilografia e pHmetria de 24 horas devem ser usados de maneira seletiva nos pacientes com sintomas atípicos, sintomas relacionados a aspiração pulmonar, evolução não satisfatória durante tratamento clínico, achados nos exames básicos de alterações motoras do esôfago ou de esvaziamento gástrico e nos estudos de recorrência da esofagite após cirurgia anti-refluxo prévia.

A técnica de Nissen modificada por videolaparoscopia para o tratamento da doença do refluxo gastroesofagiano complicada ou não, em 59 pacientes, acompanhados por um período médio de 20,8 meses, analisados por entrevista clínica, exames radiográfico, endoscópico, manométrico e cintilográfico, permitiu concluir que:

I. A técnica proposta foi realizada sem dificuldades, podendo ser concluída em todos os doentes.

2. A taxa de morbimortalidade com a técnica empregada foi baixa.

3. A técnica alcançou excelentes e bons resultados pela avaliação clínica em 93, I\% dos doentes. Quando se comparou com o diagnóstico pré-operatório de esofagite complicada e não complicada, os resultados foram $90,3 \%$ e $96,3 \%$, respectivamente, não sendo diferença estatisticamente significativa.

4. Na avaliação funcional pelo estudo radiográfico, endoscópico e manométrico, observou-se melhora nos parâmetros analisados entre o pré e o pós-operatório na quase totalidade dos pacientes, com diferença estatisticamente significativa. A cintilografia mostrou diferenças não significativas entre o pré e o pós-operatório.

\section{SUMmaRY}

\section{VIDEOLAPAROSCOPIC SURGICAL TREAT- MENT OF GASTROESOPHAGEAL REFLUX DI- SEASE: MOdIFIEd NISSEN TECHNIQUE - CLINICAL AND FUNCTIONAL RESULTS.}

BaCKGround. Gastroesophageal reflux disease is highly common worldwide and surgical treatment is being indicated more frequently. Currently, laparoscopic is the access of choice and several antireflux techniques may be used. We prefer a modified Nissen technique.

PURPOSE. To analyze preoperative clinical and functional parameters and compare with postoperative outcome of a modified valve technique performed by laparoscopy.

Methods. A group of 59 patients underwent laparoscopic modified Nissen valve. Preoperative diagnosis was made by radiological contrast exams and endoscopy in all patients. Esophagus manometry was performed in 35 patients and scintigraphy scan in 15 patients. Complicated esophagitis occurred in 54.2\%, with 21 (35.6\%) patients presenting a Barrett's epithelium. Laparoscopic surgery was performed in all patients with no conversion to open surgery, with an average time of 123.9 minutes.

REsULTS. There were no intraoperative complications. Hospital discharge occurred in an average of 47.6 hours. Symptoms as dysphagia, pain, regurgitation and flatus occurred in $48.1 \%$ of the patients in the first thirty days. Average follow-up was 20.8 months. Postoperative radiological, endoscopic, manometric and scintigrafic scan exams showed a significant improvement, as well as clinical assessment using Visick's classification, which showed excellent and good results in $93.1 \%$ of the patients.

Conclusion. Comparative analysis of clinical assessment and exam results lad us to conclude that modified Nissen surgery by laparoscopic access corrects gastroesophageal reflux in most patients followed-up. [Rev Ass Med Brasil 200 |; 47(2): |4 |-8]

KeY Words: Reflux esophagitis. Laparoscopy. Surgery.

\section{RefERÊNCIAS}

I. Collet D, Cadière Gb. Conversions and complications of laparoscopic mtreatment of gastroesophageal reflux disease. Am. J. Surg. 1995; | 69:622-26.

2. Petelin Jb. Laparoscopic approach to common duct pathology. In: Paula Al, Hashiba K, Bafutto M. eds. Cirurgia videolaparoscópica. Goiânia, Ed. Independente, 1993;93-98

3. Dallemagne B, Weerts Jm, Jehaes C. Laparoscopic Nissen fundoplication. A preliminary report. Surg. Lapar. Endosc. 1991; 1:138.

4. Geagea J. Laparoscopic Nissen's fundoplication: preliminary report on ten cases. Surg. End. 199|;5:170-73.

5. Weerts Jm, Dallemagne B, Hamoir E, et al. Laparoscopic Nissen fundoplication: detailed analysis of 132 patients. Surg. Laparosc. Endosc. 1993; 3:359-64.

6. Paula Al. Fundoplicatura total laparoscópica no tratamento cirúrgico da doença do refluxo gastroesofageano. São Paulo, 1997. (Tese Doutorado - Faculdade de Medicina da Universidade de São Paulo).

7. Paula Al, Hashiba K, Bafutto M. Tratamento laparoscópico da doença do refluxo gastroesofageano. In: Cirurgia videolaparoscópica. Goiânia, Ed. Independente, 1994;91-95.

8. Mcanena Oj, Willson Pd, Evans Df, et al. Physiological and symptomatic outcome after laparoscopic gastric fundoplication. British J. Surg. 1995;82:795-97.

9. Brandalise Na, Aranha Nc. Doença do refluxo gastroesofagiano - Técnica operatória. In: Malafaia $O$, Marchesini Jb. eds. Clínica Brasileira de Cirurgia. Colégio Brasileiro de Cirurgiões. Doença do refluxo gastroesofágico. São Paulo, Atheneu, 1996; 171-90.

10. Ferreira Eab. Esofagectomia e esofagogastroplastia por videolaparoscopia. In: Paula Al, Hashiba K, Bafutto M. eds. Cirurgia videolaparoscópica. Goiânia, Ed. Independente, 1993; 185-89.

I I. Barroso Fl. Tratamento cirúrgico da úlcera duodenal. Estado atual. In: Paula Al, Hashiba K, Bafutto M. eds. Cirurgia videolaparoscópica. Goiânia, ed. Independente, 1993; 213-16.

12. Brandalise Na, Aranha Nc. Esofagites de refluxo. Tratamento por video- laparoscopia. In: Paula Al, Hashiba K, Bafutto M. eds. Cirurgia videolaparoscópica. Goiânia, 
LOPES LR ET AL.

Ed. Independente, 1994; I I 5- I I6.

13. Savary M, Miller G. The esophagus. Handbook and atlas of endoscopy. Switzerland, Gassmann AG, 1978; 135-39.

14. Sataloff Dm, Pursnani K, Hoyo S, et al. An objective assessment of laparoscopic antireflux surgery. Am. J. Surg. 1997; 174:63-67.

15. Whiteley G, Nair R, Mccloy R. Laparoscopic gastrointestinal surgery. Scand. J. Gastroenterol. 1992; 27 Suppl. 192: |36-41.

16. Peters Jh, Heimbucher J, Kauer Wkh, et al. Clinical and physiologic comparison of laparoscopic and open nissen fundoplication. J. Am. Coll. Surg. 1995; 180:385-93.

17. Andreollo Na. Esofagite de refluxo: quando e como operar? Arq. Bras. Cir. Digest. 1996; I I (suppl):28.

18. Richardson Ws, Trus TI, Hunter Jg. Laparoscopic antireflux surgery. Surg. Clin. North Am. 1996;437-49.

19. Aranha Nc, Brandalise Na. Tratamento videolaparoscópico da esofagite de refluxo pela técnica "mista" (Nissen modificada). GED 1995; | 4:219-22.

20. Dallemagne B, Taziaux P, Weerts J, Jehaes C, MarkiewiczS. - Chirurgie laparoscopique du reflux gastro-oesophagien. Ann. Chir. 1995; 49:30-36.

21 . Juhasz Ac, Pinto Pb, Jeraldo Jcd, et al. Evaluación subjetiva y objetiva de los resultados dela cirugía antirreflujo por video laparoscopía en pacientes com reflujo gastroesofágico. Ver. Méd. Chile 1996; 124:1077-85.

22. Maclauchlan G. Oesophageal function testing and antireflux surgery. Br. J. Surg. 1996; 83: 1684-88.

23. Hinder Ra, Filipi Cj, Wetscher G, et al. Laparoscopic Nissen fundoplication is a effective treatment for gastroesophageal reflux disease. Ann. Surg. 1994:220:472-83.

24. Cattey Rp, Henry Lg, Bielefield Mr. Laparoscopic Nissen fundoplication for gastroesophageal reflux disease: clinical experience and outcome in first 100 patients. Surg. Laparosc. Endosc. 1996:6:430-33.

25. Cushieri A, Hunter J, Wolf B. Multicenter prospective evaluation of laparoscopic antireflux surgery: preliminary report. Surg. Endosc. 1993;7:505- 10.

26. Bittner Hb, Meyers Wc, Brazer Sr, Pappas TN. Laparoscopic Nissen fundoplication: operative results and short-term follw-up. Am. J. Surg. 1994; 167:193-98.

27. dallemagne B. Results of laparoscopic management of gastroesophageal reflux disease. In: Paula Al, Hashiba K, Bafutto M. eds. Cirurgia videolaparoscópica. Goiânia, Ed. Independente, 1994;89.

28. Swanstrom L, Wayne R. Spectrum of gastrointestinal symptoms after laparoscopic fundoplication. Am. J. Surg. 1994; 167:538-4I.

29. Anvari M, Allen C, Borm A. Laparoscopic Nissen fundoplication is a satisfactory alternative to long-term omeprazole therapy. British J. Surg. 1995;82:938-42.

30. Rattner Dw, Brooks Dc. Patient satisfaction following laparoscopic and open antireflux surgery. Arch. Surg. 1995; 130:289-93.

31. Deschamps C, Allen Ms, Trastek Vf, et al. Early experience and learning curve associated with laparoscopic Nissen fundoplication. J. Thorac. Cardiovasc. Surg. 1998; I | 5:28 I-84.

32. Stengel Jm, Dirado R. Laparoscopic Nissen fundoplication to treat gastroesophageal reflux. AORN Journal 1995;61:483-89.

33. Anvary M, Allen Cj. Prospective evaluation of dysphagia before and after laparoscopic Nissen fundoplication without routine division of short gastrics. Surg. Laparosc. Endosc. 1996; 6:424-29.

34. Watson Di, Reed Mwr, Johnson Ag, Stoddard Cj. Laparoscopic fundoplication for gastrooesophageal reflux. Ann. R. Coll. Surg. Engl. 1994:76:264-68.

35. Luostarinen M, Isolauri J. Effect of fundic mobilization on long-term results of Nissen fundoplication: a prospective, randomized study. In: Peracchia A, Rosati R, Bonavina L, Fumagali $U$, Bonsa S, Chella B. eds. Recent advances in diseases of the esophagus. Bologna, Monduzzi Editore, 1996; 677-80.

36. Watson Di, Pike Gk, Mathew G, et al. Prospective double blind randomised trial of laparoscopic Nissen fundoplication with division and without division of short gastric vessels. In: Peracchia A, Rosati R, Bonavina L, Fumagali U, Bonsa S, Chella B. eds. Recent advances in diseases of the esophagus. Bologna, Monduzzi Editore, 1996; 68I-86.

37. Incarbone R, Peters Jh, Heimbucher J, et al. A contemporaneous comparison of hospital charges for laparoscopic and open Nissen fundoplication. Surg. End. 1995; 9: I 5।-55.

38. Nissen R. Gastropexy as the lone procedure in the surgical repair of hiatus hernia. Am. J. Surg. 1956;92:389-92.

39. Munro W, Brancatisano R, Adams Ip, Falk Gl. Complications of laparoscopic fundoplication: the first 100 patients. Surg. Laparosc. Endosc. 1996:6:421-23.

40. Demeester Tr, Bonavina L, Albertucci M. Nissen fundoplication for gastro-esophageal reflux disease. Evaluation of primary repair in 100 consecutives patients. Ann. Surg. 1986; 204:9-40.

4I. Gama-rodrigues Jj. Hérnia hiatal por deslizamento. Esofagofundogastropexia associada à hiatoplastia - avaliação clínica, morfológica e funcional. São Paulo, 1974. (Tese - LivreDocência - Faculdade de Medicina da Universidade de São Paulo).

42. Hinder Ra, Filipi Cj. The technique of laparoscopic Nissen fundoplication. In: Paula Al, Hashiba K, Bafutto M. Eds. Cirurgia videolaparoscópica. Goiânia, Ed. Independente, 1994;85-87.

43. Hunter Jg. Laparoscopic anti-reflux surgery. In: Paula Al, Hashiba K, Bafutto M. eds. Cirurgia videolaparoscópica. Goiânia, Ed. Independente, 1993; 165.

44. Cuschieri A, Shimi S, Nathanson Lk. Laparoscopic reduction, crural repair, and fun- doplication of large hiatal hernia. Am. J. Surg. 1992; 1 63:425-30.

45. Mosnier H, Leport J, Aubert A, et al. A 270 degree laparoscopic posterior fundoplasty in the treatment of gastroesophageal reflux. J. Am. Coll. Surg. 1995; 181:220-24.

46. Cadière Gb, Himpens J, Rajan A, et al. Laparoscopic Nissen fundoplication: laparoscopic dissection technique and results. Hepatogastroenterology 1997;44:4- I0.

47. Vayre P. Le risque oesocardiotuberositaire de la cure de reflux gastro-esophagien par chirurgie coelioscopique. Chirurgie 1997; 121: 636-4.

48. Jamieson Gg, Watson Di, Britten-jones R, Mitchell Pc, Anvari M. Laparoscopic Nissen fundoplication. Ann. Surg. 1994;220: 137-45.

49. Paluzzi Mw. Laparoscopic Nissen fundoplication at a teaching center: prospective analysis of 103 consecutive patients. Surg. Laparosc. Endosc. 1997;7:363-68.

50. Laws HI, Clements Rh, Swillie Cm. A randomized, prospective comparison of the Nissen fundoplication versus the Toupet fundoplication for gastroesophageal reflux disease. Ann. Surg. 1997;225:647-53.

5I. Watson Di, Jamieson Gg, Devitt Pg, et al. Changing strategies in the performance of laparoscopic Nissen fundoplication as a result of experience with 230 operations. Surg. Endosc. 1995;9:961-66a.

52. Hallerbäck B, Glise H, Johansson B. Laparoscopic Rosetti fundoplication. Scand. J. Gastroenterol. 1995;30 Suppl 208:58-61.

53. Demeester Tr, Attwood Se, Smurck Tc, Therkildsen Dh, Hinder Ra. Surgical therapy in Barrett's esophagus. Ann. Surg. 1990; 212:528-40.

54. Montes Cg, Brandalise Na, Deliza R, Ferraz Jgp. Regression of Barrett's esophagus by antireflux surgery and bipolar electrocoagulation. Gastrointest. Endosc. 1998;47: AB73.

55. Viste A, Vindenes $H$, Gjerde S. Herniation of the stomach and necrotizing chest wall infection following laparoscopic Nissen fundoplication. Surg. Endosc. 1997; 1 1: 1029-31.

56. Hunter Jg, Trus TI, Branum Gd, Waring Jp, Wood Wc. A physiologic approach to laparoscopic fundoplication for gastroesophagea reflux disease. Ann. Surg. 1996;223: 673-85a.

57. Deschamps C, TrastekVf, Allen Ms, etal. Longterm results after reoperation for antireflux procedures. J. Thorac. Cardiovasc. Surg. 1997; I 1 3:545-50.

58. Perdikis G, Hinder Ra, Filipi Cj, et al. Laparoscopic paraesophageal hernia repair. Arch. Surg. 1997; | 32:586-89.

59. Stein Hj, Bremner Rm, Jamieson J, Demeester Tr. Effect of Nissen fundoplication on esophageal motor function. In: Paula Al, Hashiba K, Bafutto M. eds. Cirurgia videolaparoscópica. Goiânia, Ed. Independente, 1994;35-38b.

60. Mckernan Jb. Laparoscopic antireflux surgery. Int. Surg. 1994;79:342-45.

61. Mckernan Jb, Champion Jk. Laparoscopic antireflux surgery. Am. Surg. 1995; 61:530-36. 